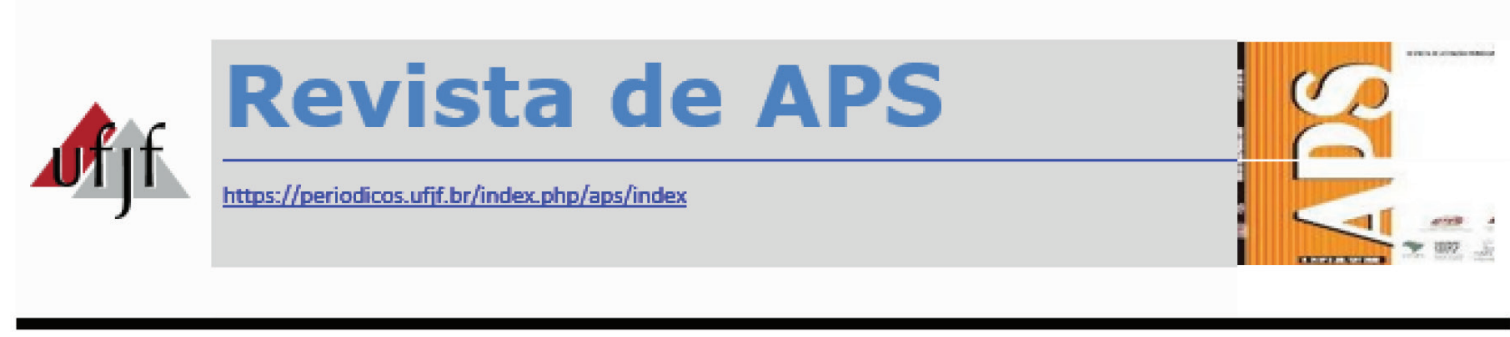

\title{
Cuidado remoto na APS: experiência do uso do celular em uma equipe de Saúde da Família de área de favela durante a crise da COVID-19
}

\section{Remote care in PHC - cell phone use experience in a Family Health team in a slum area during the COVID-19 crisis}

Luiz Montes do Amaral ${ }^{1}$, Jorge Esteves Teixeira Junior ${ }^{2}$

\section{RESUMO}

A pandemia pelo Sars-CoV2 impôs uma necessidade de reorganização do processo de trabalho das equipes de Saúde da Família, resultando em desafios ao acesso, ao manejo de condições crônicas e ao acompanhamento e monitoramento de pessoas infectadas pelo coronavírus. Este artigo descreve os usos e desafios das tecnologias chamada telefônica e WhatsApp Business em uma equipe de uma Clínica da Família em uma favela do Rio de Janeiro. Pode-se dizer que o uso foi uma experiência importante para conseguir ampliar o acesso e fortalecer vínculo, principalmente a pessoas vivendo com condições crônicas não transmissíveis e de saúde mental no cenário de restrição de acesso imposto pelo contexto da pandemia pelo Sars-CoV2.

PALAVRAS-CHAVE: Atenção Primária à Saúde. Telemedicina. Acesso aos Serviços de Saúde. População urbana.

\section{ABSTRACT}

The Sars-CoV2 pandemic imposed a need to reorganize the work process of the Family Health teams resulting in challenges to access, management of chronic conditions and monitoring of people infected with the coronavirus.

\footnotetext{
${ }^{1}$ Médico Residente pelo Programa de Residência Médica em Medicina de Família e Comunidade da Universidade Federal do Rio de Janeiro/ Escola Nacional de Saúde Pública (ENSP) - Fiocruz. E-mail: montesluiz01@gmail.com

${ }^{2}$ Médico de Família e Comunidade. Mestre em Atenção Primária à Saúde pela Universidade Federal do Rio de Janeiro.
} 
This article describes the uses and challenges of technologies like telephone calls and Whatsapp business in a Family Clinic team in a Rio de Janeiro slum. It's possible to say that this use was an important experience to expand access and strengthen bonds, especially to people living with non-transmissible chronic and mental health conditions in a scenario of restricted access due to the context of the Sars-CoV2 pandemic.

KEYWORDS: Primary health care. Telemedicine. Health services accessibility. Urban Population.

\section{INTRODUÇÃO}

A pandemia pelo novo coronavírus tem sido responsável por restrições no acesso aos serviços de saúde no mundo, forçando adaptações dos mesmos. Com o objetivo de conter a COVID-19, há a necessidade de se pensar outras formas de acesso e contato no cuidado com os usuários, ampliando o espaço das tecnologias com esa finalidade $\mathrm{e}^{1,2}$.

A Atenção Primária à Saúde (APS) é porta de entrada preferencial do sistema de saúde ${ }^{3,4}$ e a estratégia de saúde da família é cenário de coordenação do cuidado às condições crônicas de saúde ${ }^{5}$. Um importante desafio para esse nível de atenção à saúde na pandemia é a restrição de acesso, podendo acarretar complicações clínicas potencialmente evitáveis ${ }^{3,5}$. A recomendação do isolamento social e o atendimento de pessoas suspeitas da infecção da COVID-19 nas Clínicas da Família diminuiu a procura das unidades de saúde. As consultas agendadas foram em sua maioria canceladas.

Este artigo relata a experiência de implantação da tecnologia WhatsApp Business e chamadas telefônicas numa equipe de Saúde da Família no bairro de Manguinhos, Rio de Janeiro, pertencente à Clínica da Família Victor Valla (CFVV), que teve como objetivo garantir respostas às diferentes demandas em saúde da população adscrita e mitigar, na medida do possível, os efeitos negativos resultantes da restrição de acesso. O período da experiência relatada foi de março a maio de 2020.

\section{DESENVOLVIMENTO}

\section{Perfil populacional e a Clínica da Família}

A CFVV está localizada no bairro de Manguinhos, na zona norte do Rio de Janeiro, e faz parte da área programática 3.1. Com sete equipes de Saúde da Família e cerca de 15 mil pessoas cadastradas, cobre cerca de metade da população do bairro. Segundo os dados do DATA Rio ${ }^{6}$, o Índice de Desenvolvimento Social (IDS) 
de Manguinhos em 2010 foi de 0,518, abaixo da média brasileira e um dos mais baixos do município.

A equipe Renovação é uma das equipes de Saúde da Família da CFVV situada em área de favela. Com 2.475 cadastrados, a maior parte da população é do sexo feminino (55\%), entre $20-39$ anos (33\%), parda (42\%), de baixa escolaridade (39\% não completaram o ensino fundamental) e parte considerável das famílias ganha até um salário mínimo (34,3\%).

Esse perfil populacional está relacionado à grande demanda por condições agudas em saúde, mas também grande número de gestantes, crianças e portadores de doenças infecciosas crônicas, como tuberculose e HIV. A frequentação se concentra nas faixas etárias mais elevadas e portadores de doenças crônicas não transmissíveis (DCNT). Portanto, a equipe Renovação lida diariamente com a tripla carga de doenças, necessitando de um amplo arco de ofertas às diferentes demandas apresentadas.

\section{Impacto da COVID-19 e o cuidado a distância}

A pandemia de Convid-19 impôs necessárias adaptações nas ofertas por parte da unidade. Por recomendação da Secretaria Municipal de Saúde e Defesa Civil do Rio de Janeiro (SMSDC-RJ), os agendamentos e atividades de rotina ficaram restritos a grupos prioritários e pessoas com elevado risco clínico-social. Dos grupos prioritários citados pela norma técnica, destacam-se: os pacientes portadores de doenças infecciosas de importância epidemiológica, como HIV e tuberculose, acompanhamento pré-natal e pessoas com neoplasias e doenças crônicas não transmissíveis de alto risco clínico ${ }^{7}$.

Essas medidas reduziram o número de atendimentos realizados pela equipe, com risco de gerar grande demanda reprimida e possíveis agravos evitáveis em condições usuais de acesso. Identificamos que a restrição do acesso imposta pelo atual contexto se relaciona possivelmente com as diferentes fases de agravos em saúde resultantes da pandemia propostas pelo pesquisador Victor Tseng, representadas por quatro ondas ${ }^{8}$, considerando: 1a onda - mortalidade e morbidade diretamente pela COVID-19; 2 a onda - relacionada à restrição de cuidado das condições agudas não relacionadas à COVID-19; 3a onda - impacto da restrição do acesso ao cuidado de pessoas com DCNT; 4ạ onda - relacionada ao trauma físico, doença mental, estresse pós-traumático, problemas econômicos, burnout, entre outros.

No sentido de garantir respostas às demandas em saúde da população cadastrada e mitigar os efeitos do modelo supracitado no nível local em contexto de restrição de acesso presencial, a equipe Renovação iniciou uma oferta de cuidado a 
distância via celular, com ênfase nas chamadas e mensagens de texto via aplicativo WhatsApp Business.

Usamos várias das sugestões do documento "WhatsApp para Centros de Saúde - fornecendo acesso para as pessoas no século XXI" ${ }^{\prime 9}$. Para o estabelecimento de canal único de comunicação, foi criado um perfil no aplicativo citado e seu número disponibilizado para a população, que foi orientada a entrar em contato para resolução de questões administrativas, como renovação de receitas, emissão de laudos, entre outras, e questões clínicas, como o acompanhamento DCNT mais ou menos compensadas, demandas agudas, e teleorientação acerca da COVID-19.

Os médicos da equipe (médico preceptor e médico residente do segundo ano) ficaram responsáveis pelo canal, que funcionou apenas nos horários de funcionamento da unidade (segunda a sábado de $7 \mathrm{~h}-18 \mathrm{~h}$ ou até $12 \mathrm{~h}$ nos sábados), enviando respostas automáticas, dependendo do horário de funcionamento, àqueles que faziam contato. Não houve até o momento turnos exclusivos para o atendimento remoto.

A tarefa de implantação se deu com a criação interna de um guia para orientar a prática da equipe resumido no seguinte fluxograma (figura 1).

Figura 1 - Fluxograma de acesso remoto via celular

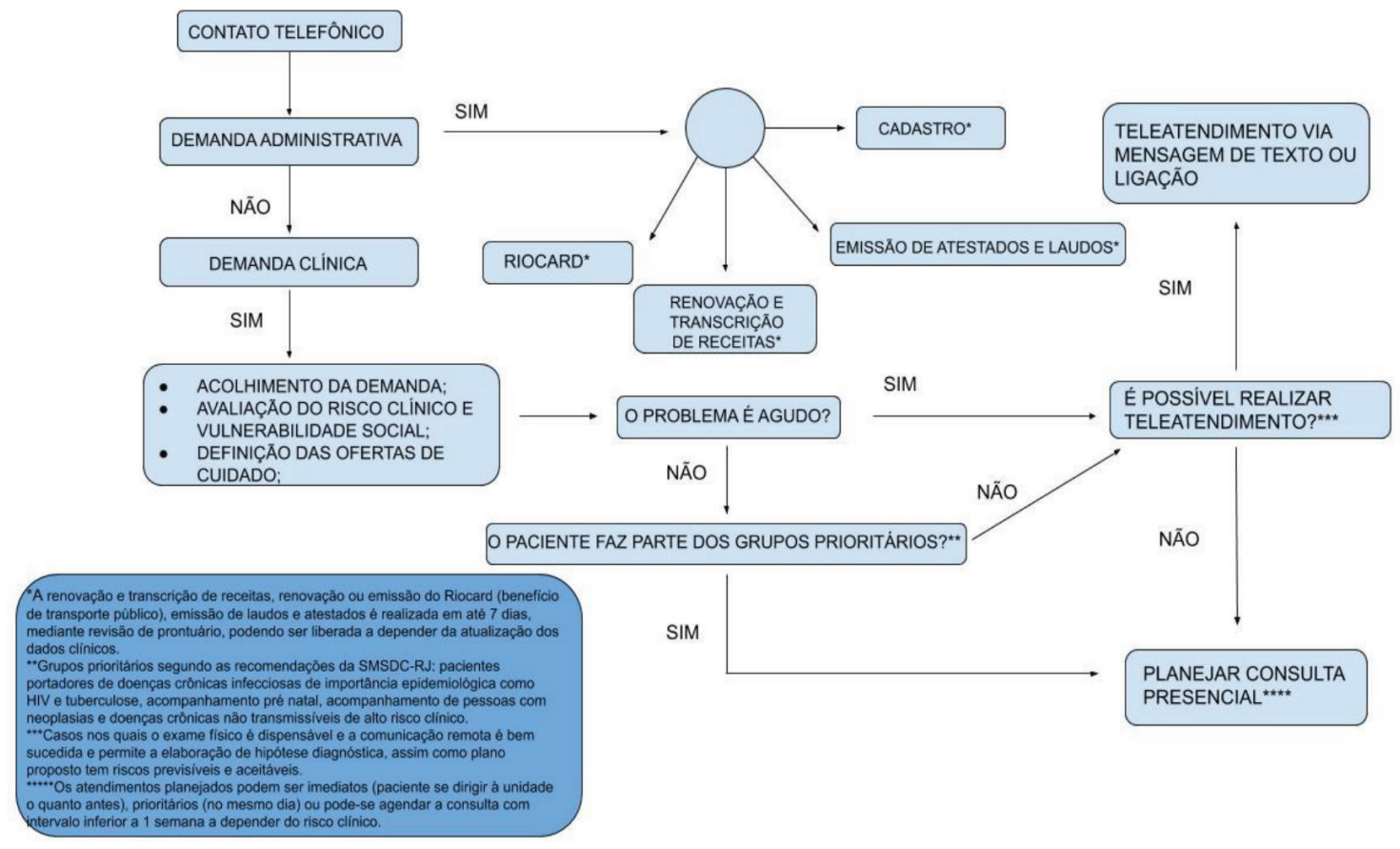

Fonte: elaborado pelos autores 


\section{Telemedicina no Brasil e mundo: potencialidades e limitações}

Telemedicina ou telessaúde pode ser definida como a utilização de recursos de tecnologia da informação e comunicação para prestar serviços de saúde a distância e compartilhar informações e conhecimento ${ }^{10,11}$. Diversos países do mundo possuem políticas públicas para esse tipo de serviço, com diferentes modelos de funcionamento ${ }^{10}$.

No Brasil, existem experiências bem-sucedidas do uso da telemedicina, especialmente, na teleconsultoria com especialidades médicas e educação permanente em saúde ${ }^{12,13}$. Anteriormente, seu uso nacional se restringia à teleconsultoria (quando há profissionais de saúde em ambos os lados da linha) e educação médica ${ }^{14}$. O PL 696/2020 ampliou o uso da telemedicina no país durante a crise da COVID-19, permitindo funções como o teleatendimento ${ }^{15}$.

A teleconsulta com o telefone celular tem sido proposta de instrumento para APS, especialmente em países desenvolvidos. Considerada uma boa alternativa às consultas presenciais, tem bons índices de satisfação por parte dos usuários e profissionais ${ }^{16-18}$, com diferentes modelos do seu uso ${ }^{18-20}$.

As principais limitações descritas na literatura sobre o tema foram a necessidade de treinamento adequado aos profissionais que farão a teleconsulta ${ }^{16,19,20}$ e a segurança do seu uso, em um ponto de vista ético/legal ou propriamente clínico (especialmente pela dificuldade de realização do exame físico) ${ }^{16-21}$. Além disso, ainda há relevante escassez de evidência de qualidade sobre o tema em relação à redução dos custos em saúde, redução do número de consultas presenciais e redução da sobrecarga de trabalho por parte dos profissionais de saúde $\mathrm{e}^{16,18,21}$.

No contexto da COVID-19, as consultas por telefone podem ser uma das estratégias no sentido de reduzir a transmissão no interior das unidades de saúde ${ }^{1,2}$. Frente à restrição do acesso presencial, o seu uso tem ampliado e torna-se potente ferramenta.

\section{Telemedicina e as favelas}

Nos países em desenvolvimento, a telemedicina possui importantes barreiras adicionais, especialmente levando-se em consideração as limitações tecnológicas, de financiamento e logísticas da operação ${ }^{11,22,23}$.

Apesar de potencialmente aumentar o acesso à saúde, o uso da telemedicina é pouco descrito nos conglomerados urbanos e nas favelas das grandes cidades. Conciliar pressão assistencial, complexidade dos casos, grande vulnerabilidade de parte da população e dificuldades de apoio de uma rede de atenção à saúde frágil na maioria das cidades brasileiras é um grande desafio para as equipes que atuam 
no contexto de favelas ${ }^{24}$ e ainda não está claro de que maneira a telemedicina pode auxiliar nesse processo.

\section{Reflexões sobre a ferramenta}

Na nossa experiência, o acesso remoto via celular foi rapidamente assimilado por parte da população cadastrada e a nosso ver bem aceito, funcionando como ferramenta na tarefa de mitigar os efeitos da pandemia da COVID-19 sobre a população coberta pela equipe.

No período estudado houve aumento progressivo de casos sintomáticos respiratórios e pacientes graves com suspeita de coronavírus e de outras condições em nossa unidade. Além deste, outro desafio relacionado ao contexto da pandemia foi o cumprimento das escalas da unidade de atendimento, necessárias durante esse período para realizar atividades como triagem de sintomáticos respiratórios, sala de exame de sintomáticos com equipamento de proteção individual (EPI) próprio, sala de observação clínica, atendimentos de urgências não relacionadas à COVID-19 e o telemonitoramento de pacientes sintomáticos respiratórios (designado a todas as equipes). Destaca-se que nesse período vários médicos foram afastados por apresentarem síndrome gripal.

Percebemos predomínio das demandas clínicas no contexto do teleatendimento, especialmente das demandas agudas em saúde. Com o uso do celular, entretanto, o acesso é facilitado e pode haver aumento dos contatos em saúde, especialmente no atual contexto, em que parte dos usuários teme ir à unidade. Isso sugere que a ferramenta pode ampliar o acesso e reduzir da demanda reprimida nesse cenário.

Sobre especificamente o acompanhamento dos pacientes com sintomas gripais, este foi feito de maneira remota, com ligações ou envio de mensagens de texto a cada 48 horas e orientações sobre sinais e sintomas de alarme. Quando não era seguro o acompanhamento, era solicitado o atendimento presencial na unidade. O telefone da Central de Atendimento da Secretaria Estadual de Saúde do Rio de Janeiro (160) foi fornecido caso não conseguissem entrar em contato com a equipe ou caso a mensagem fosse enviada fora do horário de funcionamento. Aqueles que apresentaram sinais de gravidade foram convocados à unidade ou atendidos em seu domicílio dependendo da situação e, alguns casos, orientados a entrar em contato com o Serviço de Atendimento Móvel de Urgência (SAMU).

Sobre o acompanhamento das condições crônicas não transmissíveis, tivemos satisfatório resultado e aceitação por parte da população. As principais atividades realizadas nesse sentido foram o monitoramento das medidas residenciais de pressão arterial e glicemia capilar, orientação em relação ao uso de medicamentos contínuos, 
renovação de receitas e emissão de laudos e atestados médicos, avaliação de risco e monitoramento de sinais e sintomas de alarme. Realizamos também o manejo remoto dos pacientes de saúde mental. No caso dos pacientes com transtornos mentais graves, compartilhamos este manejo remoto com o Núcleo de Apoio à Saúde da Família (NASF). Quando necessário, foi acionado o Centro de Apoio Psicossocial (CAPS) responsável pela mesma área de abrangência.

Em relação a dificuldades encontradas, percebemos limitações no uso da ferramenta. Dentre estas, destacam-se: 1) a definição dos casos que necessitavam de avaliação presencial; 2) relativo baixo acesso à internet e aparelhos celulares/computadores por parte da população, assim como a baixa escolaridade e letramento da população coberta; 3) dificuldade de inclusão dos outros profissionais, como Agentes Comunitários de Saúde e enfermeiros, no compartilhamento do cuidado remoto, considerando questões como limites profissionais e sigilos das informações.

Ainda são necessários estudos mais precisos sobre o perfil de usuários que conseguiram ou não conseguiram acessar essa ferramenta. Percebemos que alguns usuários contaram com a ajuda de familiares para fazer o contato e que alguns preferiram o uso do envio de áudios.

Consideramos que as próximas etapas para qualificação da ferramenta seriam: 1) estabelecer turnos dos profissionais mais definidos para as respostas diárias das mensagens; 2) destinar um turno ou horários para teleconsultas por videochamadas ou chamadas telefônicas; 3) elaborar estratégias para inclusão e participação de outros membros de equipe, preservando o trabalho em equipe e evitando a centralização do cuidado na figura do médico.

Sobre os rumos da telemedicina no Brasil e o uso do celular no período pós COVID-19, parece evidente que as tecnologias de comunicação (TICS) terão lugar cada vez maior na APS do país. Ainda é necessário que se estabeleçam evidências mais robustas sobre o benefício do seu uso, especialmente em países com perfil demográfico e epidemiológico semelhantes ao brasileiro e para as áreas de conglomerados urbanos e favelas. Observamos com preocupação o uso desse tipo de tecnologia para a precarização do trabalho médico. A desregulamentação do trabalho no Brasil (especialmente após a Reforma Trabalhista de 2017 - Lei Federal 13.467) e no mundo tem resultado em vínculos empregatícios precarizados que tendem à informalidade e geram insegurança trabalhista. Esse processo tem as TICs como importante motor ${ }^{25-27}$. Sobre esse tema, cunhou-se o termo "uberização" do trabalho ${ }^{26}$, que não é o mote de discussão deste trabalho, embora seja um tema de enorme relevância. 


\section{CONCLUSÃO}

A telemedicina, incluindo o uso do celular, tem sido eleita como um instrumento de diversos sistemas de saúde no mundo para a organização e ampliação do acesso e acompanhamento das DCNT. No contexto da COVID-19, em que há restrição a consultas presenciais, o seu uso desponta como uma ferramenta relativamente acessível e rápida para possibilitar respostas por parte das equipes de saúde às demandas da população adscrita.

No Brasil, a disputa acerca da regulamentação da telemedicina aparentemente será tema relevante também após o fim do contexto pandêmico, ainda que este marco seja incerto. Reflexões acerca o uso da tecnologia em áreas de conglomerados urbanos e favelas e o futuro da sua utilização na APS brasileira sugerem a necessidade de maior produção científica sobre o tema no Brasil. Seus impactos sobre o trabalho médico e o cuidado e segurança dos pacientes ainda não estão claros.

Na nossa experiência, o uso do celular no âmbito da telemedicina teve benefícios em relação ao acompanhamento dos pacientes com DCNT; às demandas agudas em saúde, o seguimento dos casos suspeitos de COVID-19, a resolutividade das questões administrativas e o fortalecimento do vínculo com a população cadastrada. Dentre as limitações do uso da ferramenta, destacam-se: a dificuldade na definição dos casos que necessitavam de avaliação presencial; a uniformização dos contatos; o registro das consultas remotas e suas repercussões ético-legais; o relativo baixo acesso à internet e aparelhos celulares/computadores e baixa escolaridade e letramento da população coberta; inexistência de turnos específicos à função; e a atenção médico-centrada.

\section{REFERÊNCIAS}

1. Greenhalgh T, Koh GCH, Car J. Covid-19: avaliação remota em Atenção Primária à Saúde. Revista Brasileira de Medicina de Família e Comunidade. 2020 abr 6; 15(42):2461.

2. Tasca R, Massuda A. Estratégias para reorganização da Rede de Atenção à Saúde em resposta à Pandemia COVID-19: a experiência do Sistema de Saúde Italiano na região de Lazio. 1. 2020 abr 15; 2(1):20-7

3. Starfield, B. Atenção primária: equilíbrio entre necessidades de saúde, serviços e tecnologia. Brasília, DF: UNESCO/MS; 2002. 
4. Giovanella L, Escorel S, Mendonça M. Porta de entrada pela atenção básica? Integração do PSF à rede de serviços de saúde. Saúde em Debate. 2003 set-dez; 27(65):278-89.

5. Vilaça E. O cuidado das condições crônicas na atenção primária à saúde: o imperativo da consolidação da estratégia da saúde da família. Brasília: Organização Pan-Americana da Saúde; 2012. 515 p.

6. Data Rio [Internet]. [citado 2020 maio 18]. Disponível em: http://www.data.rio/app/ bairros-cariocas.

7. Secretaria Municipal de Saúde do Rio de Janeiro. Nota Técnica: Orientações sobre a prevenção e manejo da transmissão e infecção pelo novo coronavírus (SARS-CoV-2) e organização dos serviços de atenção primária à saúde do município do Rio de Janeiro. Assessoria Especial de Atenção Primária à Saúde. 2020 abr.

8. Tseng V. As our friends and colleagues brave the font lines, we must also get ready for a series of aftershocks. It's very hard to plan this far ahead while we're in survival mode. We must prepare early and strategize our response to the collateral damage of \#COVID19 [Internet]. 2020 mar 30 [acesso 2020 maio 6].Twitter: @vectorsting. Disponível em: https://twitter.com/vectorsting/ status/1244671755781898241.

9. Secretaria Municipal de Saúde de Florianópolis. WhatsApp para centros de saúde: fornecendo acesso às pessoas do século XXI. Departamento de Gestão Clínica; 2019 dez.

10. Maldonado JMS de V, Marques AB, Cruz A, Maldonado JMS de V, Marques AB, Cruz A. Telemedicina: desafios à sua difusão no Brasil. Cadernos de Saúde Pública [Internet]. 2016 [citado 2020 maio 18];32. Disponível em: http://www.scielo.br/scielo. php?script=sci_abstract\&pid=S0102-311X2016001402005\&Ing=en\&nrm=iso\&tlng=pt.

11. Celes RS, Rossi TRA, de Barros SG, Santos CML, Cardoso C. A telessaúde como estratégia de resposta do Estado: revisão sistemática. Rev Panam Salud Publica [Internet]. 2018 ago 10 [citado 2020 maio 18]; 42. Disponível em: https://www.ncbi. nlm.nih.gov/pmc/articles/PMC6385855/12.

12. Eno DCF. Telessaúde no apoio a médicos da atenção primária [tese de doutorado]. Porto Alegre: Universidade Federal do Rio Grande do Sul; 2011. 110 p.

13. Alkmim MB, Figueira RM, Marcolino MS, Cardoso CS, Pena de Abreu M, Cunha LR, et al. Improving patient access to specialized health care: the Telehealth Network of Minas Gerais, Brazil. Bull World Health Organ. 2012 maio 1; 90(5):373-8.

14. Catapan S de C, Calvo MCM, Catapan S de C, Calvo MCM. Teleconsulta: uma Revisão Integrativa da Interação Médico-Paciente Mediada pela Tecnologia. Revista Brasileira de Educação Médica [Internet]. 2020 [citado 2020 maio 18]; 44(1). 
Disponível em: http://www.scielo.br/scielo.php?script=sci_abstract\&pid=S010055022020000100304\&lng=en\&nrm=iso\&tlng=pt.

15. Agência Senado. Senado aprova uso da telemedicina durante pandemia de covid-19 [Internet]. Senado Federal. [citado 2020 maio 18]. Disponível em: https:// www12.senado.leg.br/noticias/materias/2020/03/31/senado-aprova-uso-datelemedicina-durante-pandemia-de-covid-19.

16. Galen LS van, Car J. Telephone consultations. BMJ [Internet]. 2018 mar 29 [citado 2020 maio 18]; 360. Disponível em: https://www.bmj.com/content/360/bmj.k1047.

17. Khan MNB. Telephone consultations in primary care, how to improve their safety, effectiveness and quality. BMJ Qual Improv Rep [Internet]. 2013 out 1 [citado 2020 maio 18]; 2(1). Disponível em: https://www.ncbi.nlm.nih.gov/pmc/articles/ PMC4652702/.

18. Downes MJ, Mervin MC, Byrnes JM, Scuffham PA. Telephone consultations for general practice: a systematic review. Systematic Reviews. 2017 jul 3; 6(1):128.

19. McKinstry B, Watson P, Pinnock $H$, Heaney D, Sheikh A. Telephone consulting in primary care: a triangulated qualitative study of patients and providers. Br J Gen Pract. 2009 jun; 59(563):e209-18.

20. Greenhalgh T, Vijayaraghavan S, Wherton J, Shaw S, Byrne E, Campbell-Richards D, et al. Virtual online consultations: advantages and limitations (VOCAL) study. BMJ Open. 2016 jan 1; 6(1):e009388.

21. Bunn F, Byrne G, Kendall S. Telephone consultation and triage: effects on health care use and patient satisfaction. Cochrane Database Syst Rev. 2004 out 18; (4):CD004180.

22. Bali S. Barriers to Development of Telemedicine in Developing Countries. Telehealth [Internet]. 2018 dez 5 [citado 2020 maio 18]. Disponível em: https://www. intechopen.com/books/telehealth/barriers-to-development-of-telemedicine-indeveloping-countries.

23. Combi C, Pozzani G, Pozzi G. Telemedicine for Developing Countries. A Survey and Some Design Issues. Appl Clin Inform. 2016; 7(4):1025-50.

24. Nunes M, Stelet BP, Esteves J. Favela. Tratado de Medicina de Família e Comunidade. 2a edição. Porto Alegre, Brasil: Artmed; 2019. P 490-97.

25. Pinheiro SSM, Souza $M$ de $P$, Guimarães KC. Uberização: a precarização do trabalho no capitalismo contemporâneo. Serviço Social em Debate [Internet]. 2020 abr 6 [citado 2020 maio 18]; 1(2). Disponível em: http://revista.uemg.br/index.php/ServSoc-Debate/article/view/3923.

26. Abilio LC. Uberização: Do empreendedorismo para o autogerenciamento subordinado. Psicoperspectivas. 2019 nov; 18(3):41-51. 
27. Antunes R. O privilégio da servidão: novo proletariado de serviços na era digital. 1a edição. São Paulo: Boitempo; 2018. p. 65-81. Cap. 3: Infoproletariado, informalidade, (i)materialidade e valor.

Submissão: maio de 2020.

Aprovação: outubro de 2020. 